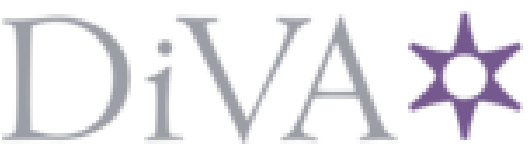

http://www.diva-portal.org

Preprint

This is the submitted version of a paper presented at 9th IAPR International Conference on Biometrics, Halmstad, Sweden, June 13-16, 2016.

Citation for the original published paper:

Gangwar, A., Joshi, A., Singh, A., Alonso-Fernandez, F., Bigun, J. (2016)

IrisSeg: A Fast and Robust Iris Segmentation Framework for Non-Ideal Iris Images.

In:

N.B. When citing this work, cite the original published paper.

Permanent link to this version:

http://urn.kb.se/resolve?urn=urn:nbn:se:hh:diva-31745 


\section{IrisSeg: A Fast and Robust Iris Segmentation Framework for Non-Ideal Iris Images}

\author{
Abhishek Gangwar, Akanksha Joshi, Ashutosh \\ Singh \\ Centre for Development of advanced \\ Computing (CDAC), Mumbai, India \\ \{abhishek, akanksha, ashutoshsingh\} @cdac.in
}

\author{
Fernando Alonso-Fernandez, Josef Bigun \\ Halmstad University, \\ Box 823. SE 301-18 Halmstad, Sweden \\ \{feralo, Josef.Bigun\} @hh. se
}

\begin{abstract}
This paper presents a state-of-the-art iris segmentation framework specifically for non-ideal irises. The framework adopts coarse-to-fine strategy to localize different boundaries. In the approach, pupil is coarsely detected using an iterative search method exploiting dynamic thresholding and multiple local cues. The limbic boundary is first approximated in polar space using adaptive filters and then refined in Cartesian space. The framework is quite robust and unlike the previously reported works, does not require tuning of parameters for different databases. The segmentation accuracy (SA) is evaluated using well known measures; precision, recall and F-measure, using the publicly available ground truth data for challenging iris databases; CASIAV4-Interval, ND-IRIS-0405, and IITD. In addition, the approach is also evaluated on highly challenging periocular images of FOCS database. The validity of proposed framework is also ascertained by providing comprehensive comparisons with classical approaches as well as state-of-the-art methods such as; CAHT, WAHET, IFFP, GST and Osiris v4.1. The results demonstrate that our approach provides significant improvements in segmentation accuracy as well as in recognition performance that too with lower computational complexity.
\end{abstract}

\section{Introduction}

Iris is considered one of the most accurate biometrics because of its stability over long time, non-invasiveness, and unique pattern [1]. The accuracy of iris localization, decides the iris texture to be used for feature extraction and thus accuracy of overall recognition system, which makes this stage an important part of iris recognition. Daugman's Integro-Differential Operator (IDO) [1] and Wilde's circular Hough transform based approach [2] are the most significant and well known techniques for iris segmentation. Based on the basic steps in these two methods, many techniques have been proposed with some variations $[5,7,9,14]$. These classical and other similar approaches achieve excellent accuracy when iris images are of high-quality. However, in real world applications images may contain some types of challenges such as, occlusion, illumination variation, specular reflections, pupil constriction/expansion, image resolution, etc.. The prior art also discloses that almost all of such methods assume that pupillary and limbic boundaries are circular/ elliptical $[1,2,14,18]$. Recently, researchers discovered that in non-ideal iris images and even in ideal images, some shapes of pupil and iris are not perfect circle or ellipse [11]. Also, sometimes in non-ideal images, gradient on pupillary, iris and eyelid boundaries is not sufficient enough for these models. Thus, performance of such methods degrades significantly in non-ideal images. The other problems are that, these methods are time taking and computationally intensive due to brute-force approach and search of large parameter space. Moreover, parameters which are set to work for one database, may not work for other database. To address these issues, recently, researchers have started focusing towards non-ideal iris segmentation and various methods have been proposed [3-6,10-12,15-17] including adaptation of different types of active contours [11-13,19]. The active contours require the initial boundary from where it can proceed to localize actual boundary and a large number of iterations is a mandatory requirement, which makes the whole process a bit complex. For a detailed discussion on iris segmentation approaches and current issues, we refer the readers [7].

In this paper, we propose an iris segmentation framework ${ }^{1}$ which is simple to implement, robust, can work with non-ideal iris images without requirement of database specific parameter tuning. The major contributions include-

- Highly effective pupil detection module based on a Pupil Candidate Bank (PCB) created using iterative thresholding followed by selection of best candidate by exploiting multiple local features.

- Coarse approximation of limbic boundary in polar space using adaptive filters followed by dual circle based refinement of limbic boundary in angular image.

- Eyelid boundary detection based on adaptive and similarity based filters and boundary refinement based on morphological operation with arched kernels, followed by final boundary estimation using parabola fitting.

The rest of the paper is organized as follows. Section 2

${ }^{1}$ Code (in Matlab) is available at https://github.com/cdac-cvml/IrisSeg/ 


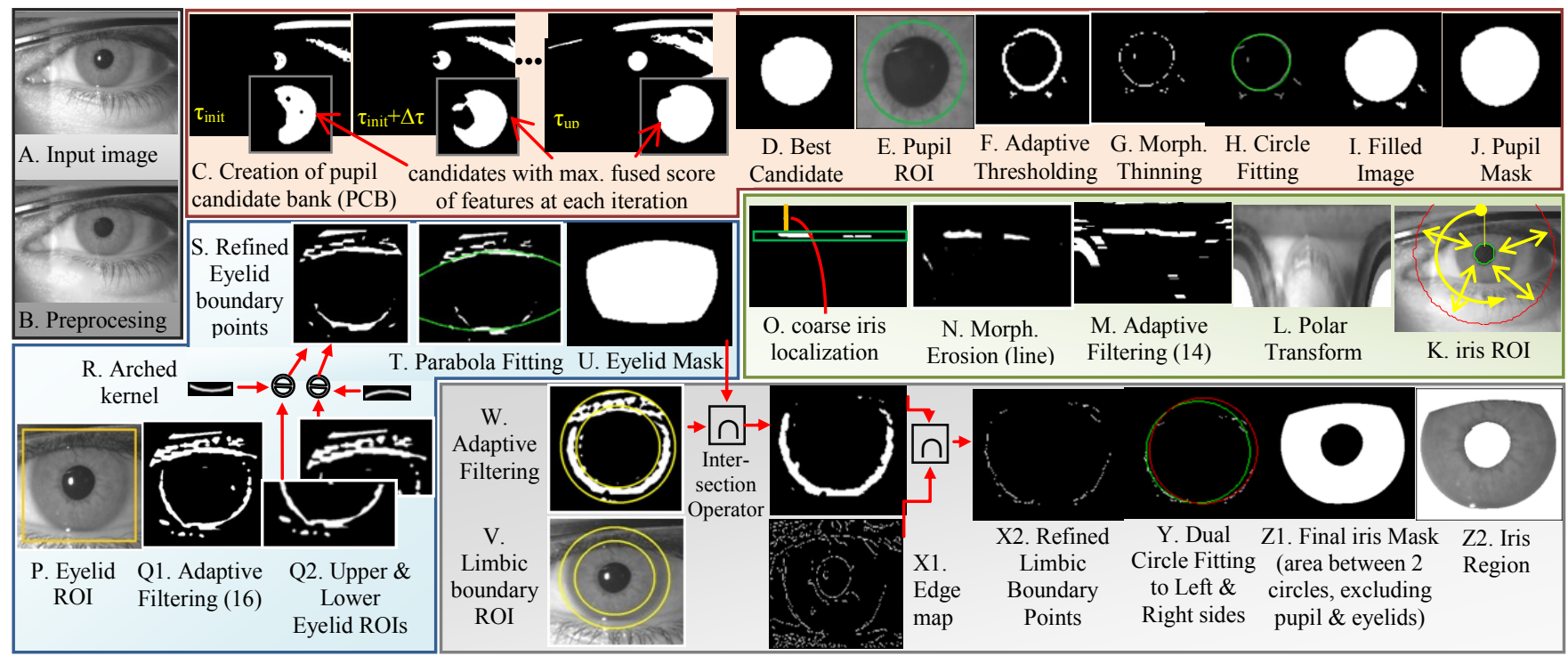

Figure 1:Illustration of Proposed Framework; (C) Construction of PCB; (D) best candidate selection using features i.e. $\mathrm{F}_{\mathrm{RR}}, \mathrm{F}_{\mathrm{RMI}}, \mathrm{F}_{\mathrm{GMD}}, \mathrm{F}_{\mathrm{CV}}$; (E-J) Refinement of pupil mask; (K-O) coarse iris localization using adaptive filters; (P-U) eyelid localization using adaptive filters, arched kernels and parabola fitting; (V-Z) refinement of iris mask.

explains proposed segmentation framework. Experimental analysis is given in section 3 and conclusion in section 4.

\section{Proposed Framework for iris Segmentation}

An overview of proposed iris segmentation framework is shown in Fig. 1. It consists of a coarse-to-fine strategy and adaptive computation of the parameters for enhanced iris segmentation to cope with bad quality factors such as scaling, translation, uneven illumination, irregular shape etc.. The framework exploits the mandatory geometrical and textural cues of pupil and iris contours to obtain superior results. For all the images in this paper, the upper left corner is taken as the origin of coordinate system. Various components of the framework are as follows-

\subsection{Preprocesing}

Let $\{\mathrm{I}(\mathrm{x}, \mathrm{y}) \mid 1 \leq \mathrm{x} \leq \mathrm{N}, 1 \leq \mathrm{y} \leq \mathrm{M}\}$, represents an input image with gray values within interval [0,1]. During preprocesing we performed removal of specular reflection and smoothing of image.

A. Treatment of Specular Reflections; To remove specular reflections, a mask is created, which contains pixels susceptible to specular reflection given as$\operatorname{Mask}_{\mathrm{sr}}(\mathrm{x}, \mathrm{y})=\mathrm{I}(\mathrm{x}, \mathrm{y}) \geq \tau_{\mathrm{sr}}$. To compute threshold $\tau_{\mathrm{sr}}$, the input image $\mathrm{I}(\mathrm{x}, \mathrm{y})$ is divided into 4 -by-4 blocks. For each block, the average is computed and highest average value is taken as threshold $\tau_{\mathrm{sr}}$. The reflection regions in $\operatorname{Mask}_{\mathrm{sr}}(\mathrm{x}, \mathrm{y})$ are treated by applying inpainting procedure and are filled by interpolating inward from the pixel values on region boundary by solving Laplace's equation.

B. Smoothing; in order to smoothen the image, we adopted "Median Filter" ([8,8]). The median filtering makes image more uniform and removes the effect of iris texture and eyelashes up to certain level without reducing the sharpness of the image, which helps in segmentation.

\subsection{Pupil Segmentation}

Our pupil detection approach comprises of a number of steps, including; A) construction of "Pupil Candidate Bank" (PCB) containing objects obtained through a dynamic and iterative thresholding process, B) selection of optimal pupil candidate in PCB using our proposed "Pupil Selection Features" C) refinement of pupil boundary.

A. Construction of "Pupil Candidate Bank" (PCB); in our approach, the image $\mathrm{I}(\mathrm{x}, \mathrm{y})$ is binarized iteratively for a range of thresholds in the interval- $\left[\tau_{\text {init }}, \tau_{u p}\right]$. During each iteration, the binary image is scanned pixel-by-pixel and pixels are grouped into components using 8-connectivity. The very small objects are removed and remaining components are labeled by assigning each one a unique label $\{\mathrm{i} \mid \mathrm{i}=1,2, . ., \mathrm{n}\}$. To compute the properties of these labeled binary candidates, we create separate binary image (M-by-N) for each of them by assigning value 1 to pixels with the specified label and 0 elsewhere. Let $\boldsymbol{S}=$ $\left\{\boldsymbol{s}_{1}, \boldsymbol{s}_{2}, \ldots, \boldsymbol{s}_{\boldsymbol{n}}\right\}$ be the set of these binary object images that may contain pupil. To compute initial threshold $\left(\tau_{\text {init }}\right)$, the image is divided into blocks of b-by-b size and average for each block is calculated. The lowest average value is considered as initial threshold value $\left(\tau_{\text {init }}\right)$. We empirically determined that a block of $b=8$ (i.e. 8 -by- 8 pixels) provides the optimal trade-off between accuracy and computing performance.

B. Proposed Local Features for Pupil Candidate Selection; After obtaining binary images, our goal is to 
distinguish optimal pupil candidate with other candidates. To evaluate each candidate, we considered few assumptions such as; i) compared to other dark candidates in eye image, pupil is relatively more round, ii) average intensity of pupil region is lowest in the image, iii) pupil region can be considered relatively more homogeneous than other darker structures, iv) spatial frequency of iris region is significantly higher than pupil region. Following these assumptions, we have developed four local features to separate pupil candidate from other objects.

i. Region Roundness Feature $\left(\mathbf{F}_{\mathbf{R R}}\right)$; The proposed roundness feature $\left(F_{R R}\right)$ measures the roundness of the binary candidate in $S_{i}$. The feature can be defined as -

$$
F_{R R}^{i}=\frac{\sqrt{A_{i} / \pi}}{\text { semi-majoraxis }_{i}}
$$

Where, semi-majoraxis $i_{i}$ is the longest radius of $i^{\text {th }}$ object ellipse and $A_{i}$ provides the area of the $i^{\text {th }}$ object in pixels and is calculated as -

$$
A_{i}=\sum_{x=1}^{N} \sum_{y=1}^{M} s_{i}(x, y)
$$

Here, roundness measure is motivated by the fact that circular object will have a roundness value close to 1 and other shapes will take comparatively a smaller value.

ii) Region Mean Intensity Feature $\left(\mathbf{F}_{\mathbf{R M I}}\right)$; This cue measures the general brightness of the region in image corresponding to binary candidate $\mathrm{s}_{\mathrm{i}}$. It can be defined as-

$$
F_{R M I}^{i}=\frac{1}{A_{i}} \sum_{x=1}^{N} \sum_{y=1}^{M} I(x, y) \mid s_{i}(x, y)=1
$$

This feature is motivated by the fact that pupil region in eye image takes relatively lower average pixel intensity.

iii) Gradient Magnitude Density Feature $\left(\mathbf{F}_{\mathrm{GMD}}\right)$; The number of pixels with high gradient in a given region shows some indication of the busyness of that region. The value will be lower in the regions with uniform distribution than that of changing patterns. To exploit this information, we apply 2-D spatial gradient filter to the region in $\mathrm{I}(\mathrm{x}, \mathrm{y})$ corresponding to binary candidate of $s_{i}$, and comput the gradient magnitude, $\operatorname{Gmag}(\mathrm{x}, \mathrm{y})$ at each pixel followed by a threshold operation to detect the extreme values of the gradient which correspond to edge pixels. The proposed feature (high gradient pixels (i.e. edge pixels) per unit area) can be computed as-

$$
F_{G M D}^{i}=\frac{1}{A_{i}} \sum_{x=1}^{N} \sum_{y=1}^{M} s_{i}(x, y) \mid \operatorname{Gmag}(x, y) \geq \tau
$$

Where, $\tau$ is some threshold and Gmag is calculated as-

$\left(\begin{array}{lll}\mathrm{z}_{1} & \mathrm{z}_{2} & \mathrm{z}_{3} \\ \mathrm{z}_{4} & \mathbf{z} & \mathrm{z}_{6} \\ \mathrm{z}_{7} & \mathrm{z}_{8} & \mathrm{z}_{9}\end{array}\right)$

$$
\begin{gathered}
\operatorname{Gmag}(\mathrm{z})|=|\left(\mathrm{z}_{1}+2 \mathrm{z}_{2}+\mathrm{z}_{3}\right)-\left(\mathrm{z}_{7}+2 \mathrm{z}_{8}+\mathrm{z}_{9}\right) \mid \\
+\left|\left(\mathrm{z}_{3}+2 \mathrm{z}_{6}+\mathrm{z}_{9}\right)-\left(\mathrm{z}_{1}+2 \mathrm{z}_{4}+\mathrm{z}_{7}\right)\right|
\end{gathered}
$$

The Gmag function of (5) provides an approximate magnitude similar to Sobel edge descriptor but in a much faster way.

iv) Contextual Variation Feature $\left(\mathbf{F}_{\mathbf{C V}}\right)$; The pupil and iris regions exhibit different intensity distribution and pattern. The proposed, $\mathrm{F}_{\mathrm{CV}}$ feature exploits the mandatory adjacency property of pupil and iris, and difference in their texture, which provides discriminative property for the pupil from other misleading artifacts. To compute this feature, we compute candidate's approximate radius $\left(\mathrm{r}_{\mathrm{i}}\right)$ and center of mass $\left(C x_{i}, C y_{i}\right)$. Then, we cut a small horizontal rectangle region from input image passing through centroid of the candidate as, strip $_{i}=$ $\left\{I(x, y) \mid C x_{i}-2 r_{i} \geq x \leq C x_{i}+2 r_{i}, C y_{i}-r_{i} / 2 \geq y \leq\right.$ $\left.C y_{i}+r_{i} / 2\right\}$. Let $\sigma_{\text {in }}$ and $\sigma_{\text {out }}$ are standard deviations of the inner and outer regions in $\operatorname{strip}_{i}$ respectively, then the proposed feature can be defined as-

$$
F_{C V}^{i}=\sigma_{\text {in }}^{i} / \sigma_{\text {out }}^{i}
$$

C. Optimal Pupil Candidate Selection; In order to achieve a final decision, the scores outputted by these features are consolidated and each candidate obtained during various iterations is represented by a feature vector comprising of all the features, given as $F_{i}=\left[F_{R R}^{i}, F_{R M I}^{i}, F_{G M D}^{i}, F_{C V}^{i}\right]$. To compute resulting output score $\left(\delta_{\mathrm{i}}\right)$, the proposed measures are combined as-

$$
\delta_{i}=\varphi\left(F_{R R}^{i}\right) \cdot \phi\left(F_{R M I}^{i}\right) \cdot \psi\left(F_{G M D}^{i}\right) \cdot \lambda\left(F_{C V}^{i}\right)
$$

Where, $\varphi(), \phi(), \psi()$, and $\lambda()$ are normalization functions. The roundness measure, $F_{R R}^{i} \in(0,1]$, provides the most discriminating information, hence taken on exponential scale, given as-

$$
\varphi(x)=\mathrm{e}^{x}-1
$$

The contribution of $F_{R M I}^{i}$, is standardized as -

$$
\phi(x)=1-x^{2}
$$

The relationship between Gradient Magnitude Density Feature and pupil candidate selection is also found non-linear and $\psi$ is computed as-

$$
\psi(x)=1-0.05 * e^{5 x}
$$

To standardize $F_{C T V}^{i}$ measure, $\lambda$ function is given as-

$$
\lambda(x)=\left\{\begin{array}{l}
1+\epsilon-x, \text { if } x \leq 1 \\
\epsilon \quad \text { otherwise }
\end{array}\right.
$$

Finally, the candidate having highest value for fused score $\left(\delta_{\mathrm{i}}\right)$ is selected as optimal pupil candidate and the selected candidate is used as initial pupil mask,

$$
\operatorname{Mask}_{p}^{\text {init }}=s_{i}(x, y) \mid \delta_{\mathrm{i}}=\max \left(\delta_{1}, \delta_{2}, \ldots, \delta_{n}\right)
$$

The selected candidate can be described with center of mass and radius $\left(C p x, C p y, r_{p}\right)$. From the aforementioned discussion it can be observed that, i) the proposed features exploit different characteristics of the objects. ii) Since, the proposed features are based on relative local physical properties of the image, they generalize well for different databases, iii) the proposed features show different distribution for true pupil and non pupil candidates.

D. Refinement of the Pupil Boundary; The pupil, detected in previous step is based on global thresholding, thus there may be some localization inaccuracies. Hence, further boundary refinement is required to detect sufficient valid pupil-iris boundary points, specifically, for the non-ideal images. The most of the traditional and widely used Integro-Differential Operator, circular Hough 
Transform, and cubic smoothing spline based methods too fail to localize exact pupil boundary points in non-ideal images. To identify proper pupil boundary points, we create an ROI image $h_{p}(x, y)$ of size M-by-N from image $\mathrm{I}(\mathrm{x}, \mathrm{y})$. In $h_{p}$, taking $(C p x, C p y)$ as center, all the pixels outside a circular search region of radius $r_{s p}=1.5 \times r_{p}$ are set to 0 (Fig. 1(E)). Next, we statistically examine the intensity characteristics in the search region and threshold each pixel adaptively to accommodate changing lighting conditions in the image, e.g. those occurring as a result of a strong illumination gradient or shadows. For each pixel, the value of threshold is measured using the average of the gray values in a w-by-w neighborhood. If the pixel is significantly darker than the average, it is assigned a value 1 otherwise it is assigned 0 . The pupil boundary mask $\left(B_{p}\right)$ from $h_{p}$ is obtained as follows-

$$
B_{p}(x, y)=\left\{\begin{array}{cc}
1, & \text { if } h_{p}(x, y)<\mu_{w}(x, y)-\Delta c \\
0 & \text { otherwise }
\end{array}\right.
$$

Where, $\mu_{w}(x, y)$ computes the mean of gray values in a $\mathrm{w}$-by-w neighborhood around the center point $(\mathrm{x}, \mathrm{y})$ and $\Delta c$ is a constant. The use of local mean makes the process adaptive. The parameters $\mathrm{w}=10$, and $\Delta c=5$ are found appropriate for all datasets (Fig. 1(F)).

The method highlights (i.e. sets 1) the darker pixels in the area near pupillary boundary because on boundary regions the mean value lies between the intensity values of foreground and background and, therefore, separates easily. In the uniform regions however, the range of intensity values within a local neighborhood is very small and their mean is close to the value of the center pixel. With use of $\Delta \mathrm{c}$, all pixels which exist in a uniform neighborhood are set to background (0).

Next, to obtain full pupil contour, we fill the region inside pupil boundary of $B_{p}(x, y)$. For that, morphological thinning is done on $B_{p}(x, y)$ followed by circle fitting (Fig. $1(\mathrm{G}-\mathrm{H})$. An intermediate image $B_{p}^{2}$ is obtained by filling region inside this circle with value $1 . B_{p}^{2}$ is then merged with $B_{p}$ (Fig. 1(I)) and object having largest area is retained. To further compensate for irregularities on this object's boundary, an iterative morphological erosion process is performed. The erosion breaks thin connections and smoothes the boundary. To compensate for erosion, we perform morphological dilation, thus facilitating final pupil mask $\operatorname{Mask}_{p}\left(\right.$ Fig. 1(J)). Mask ${ }_{p}$ basically represents better pupil boundary compared to initial mask because it contains adaptively thresholded boundary pixels, whereas Mask $_{p}^{\text {init }}$ contains globally thresholded pixels.

\subsection{Coarse Iris localization}

Once the parameters for pupil are known, the next step is to localize limbic boundary. Most of the published approaches are based on finding iris outer boundary using image information in radial direction $[1,2,3,12,17]$, whereas, our approach first estimates it by transforming the image into polar space, which transforms the near circular iris contour into near linear structure. This makes the localization process computationally less expensive, as well as we also achieved better localization accuracy. The average radius of iris can vary from 1 to 8 times of the pupil radius, using this information a region $\mathrm{R}_{1}$ (Fig. $1(\mathrm{~K})$ ) around pupil is unwrapped with respect to the pupil center and converted into polar domain. The region $\mathrm{R}_{1}$ can be defined as- $R_{1}=\{(x, y) \mid x=C p x+r \cos \theta, y=C p y+$ $r \sin \theta, r_{p} \leq r \leq 8 * r_{p}, \theta \in[1,360]$. A sample unwrapped polar image $\mathrm{I}_{\text {polar }}(\mathrm{r}, \theta)$ containing iris region is shown in Fig. 1(L). Next, the pixels in $I_{\text {polar }}$ are thresholded adaptively to highlight limbic boundary candidate pixels. The thresholded image ( $\left.\mathrm{I}_{\text {polar }}^{1}\right)$ is computed as-

$I_{\text {polar }}^{1}(x, y)=\left\{\begin{array}{c}1, \text { if } I_{\text {polar }}(x, y)<\mu_{w}(x, y)-\Delta c, \\ \text { and, } \operatorname{Var}_{w 1}(x, y)>\eta . \operatorname{Var}_{w 2}(x, y) \\ 0 \quad \text { otherwise }\end{array}\right.$

Where, $\mu_{w}(x, y), \Delta c$ are as given in (13), $\Delta c=5, \mathrm{w}=15$, and $\eta=1.5$ are found satisfactory. $\operatorname{Var}_{w 1}(x, y)$ and $\operatorname{Var}_{w 2}(x, y)$ compute variance of gray values in a window $\mathrm{w} 1=\{(\mathrm{x}, \mathrm{y}) \mid \mathrm{x} \in[\mathrm{x}-4, \mathrm{x}+4], \mathrm{y} \in[\mathrm{y}-16, \mathrm{y}]\}$ above the point $(\mathrm{x}, \mathrm{y})$ and, $w 2=\{(x, y) \mid x \in[x-4, x+4], y \in$ $[y, y+16]\}$ below point $(\mathrm{x}, \mathrm{y})$, respectively. An image obtained using (14) is shown in Fig. 1(M).

In order to further refine the candidate iris boundary pixels and to filter out other artifacts, we applied morphological closing with line structuring element $\mathrm{S}_{\text {line }}$, defined as $I_{\text {polar }}^{2}=I_{\text {polar }}^{1} \bullet S_{\text {line }}$. This step is motivated by the fact that, morphological processing with line structuring element pursues the goals of excluding the non-linear structures while preserving linear structures of sufficient size (Fig.1 N). Sometimes, unwanted upper and lower eyelids may also survive this morphological processing, thus pixels in these regions are set to 0 . The regions corresponding to upper and lower eyelids are taken as; $R_{u p}=\{(x, y) \mid 1<x \leq 60,300<x \leq 360\}$ and $R_{\text {lower }}=$ $\{(x, y) \mid 120<x \leq 240\}$. Next, the image is scanned at each position from top to bottom, moving a rectangular window (w-by-N) pixels. The window position with maximum number of white pixels (Fig $1(\mathrm{O})$ ) is considered as limbic boundary, defined as-

$r_{r i}=\underset{i \in \mathbb{Z}}{\operatorname{argmax}}\left(\sum_{x=1}^{N} \sum_{y=i}^{i+w} I_{\text {polar }}^{1}(x, y) \mid 1 \leq i \leq M-w\right)$

Where $\mathrm{M}$ and $\mathrm{N}$ are height and width of image and $\mathrm{w}=7$ is taken during experimental evaluation.

The computed value of $r_{r i}$ gives a good estimate of iris width, however, it may not be valid to consider the value of $r_{r i}$ to represent optimal limbic boundary due to; a) some iris images may not be proper circular, thus complete boundary pixel population may not form completely linear structure, b) $\mathrm{I}_{\text {polar }}$ is created with respect to pupil center, however, 
the iris and pupil circumferences may not be concentric. This coarse estimation of limbic boundary can be described using parameters as- $\left(C p x, C p y, r_{r i}\right)$.

\subsection{Estimation of eyelids occlusion}

The accurate identification of pixel population damaged by eyelids occlusion is a crucial step for high accuracy but it is highly challenging because of shape irregularity of eyelids and texture variations due to eyelashes. Most of the procedures in literature approximate eyelid directly either by using parabola fitting [1,2] or line fitting [9]. The line fitting methods are relatively faster, but are susceptible to miss some genuine iris pattern or to include part of eyelid pattern in the template. Some model based approaches have also been proposed [4,5] but, they face generalization issues and cannot be effectively adopted to different types of databases. Most of these methods require large search space, making process computationally intensive.

Our proposed method for eyelid boundary detection consists in a two-tier analysis. In first tier of the approach, proper eyelid candidate pixels are detected by generating edgemap and applying multistep eyelid candidate pixel refinement procedure. Next, in second tier, eyelid modeling is performed by applying a parabola fitting procedure.

The process starts by computing an image $E^{\text {roi }}$ of same size as $\mathrm{I}(\mathrm{x}, \mathrm{y})$, which contains eyelid ROI region and pixels outside eyelid region are all $0 \mathrm{~s}$. The $E^{\text {roi }}$ initialized with all $0 \mathrm{~s}$ is obtained as- $E^{r o i}(x, y)=\{I(x, y) \mid C p x-\gamma<x<$ $C p x+\gamma, C p y-\gamma<y<C p y+\gamma\}$ and $\gamma=1.2 * r_{r i}+$ $r_{p}$. We then apply 1-D horizontal rank filter on $E^{r o i}$ resulting into $E_{\text {rank }}^{r o i}$ to reduce the impact of vertical, dark eyelashes. The rank order filter computes the $\mathrm{p}^{\text {th }}$ percentile of pixels on a w sized window around each point. Experimentally, we found that, $\mathrm{w}=9$ and $\mathrm{p}=3$ provide satisfactory results.

Next, pixels in $E_{\text {rank }}^{\text {roi }}$ are filtered adaptively to highlight eyelids edge pixels using a very efficient method developed by us and the filtered image $\left(E_{\text {adapt }}^{\text {roi }}\right)$ is obtained as$E_{\text {adapt }}^{\text {roi }}(x, y)$

$=\left\{\begin{array}{c}1, \quad \text { if } \quad E_{\text {rank }}^{\text {roi }}(x, y)<\mu_{w}(x, y)-\Delta c, \quad \text { and } \\ D\left(P_{w}(x, y), P_{w}^{\text {ref } 1}\right)>\kappa \cdot D\left(P_{w}^{\text {ref } 2}, P_{w}^{\text {ref } 1}\right) \\ 0 \quad \text { otherwise }\end{array}\right.$

Where, $\mu_{w}(x, y), \Delta c$ are similar to $(13), P_{w}(x, y)$ is a w-by-w patch around the center point $(\mathrm{x}, \mathrm{y}), P_{w}^{\text {ref } 1}$ and $P_{w}^{r e f 2}$ are two w-by-w reference patches from left and right iris regions respectively, which are least prone to noise. $P_{w}^{r e f 1}$ and $P_{w}^{r e f 2}$ are taken as- $P_{w}^{r e f 1}=\{I(x, y) \mid C p x+$ $\gamma-w / 2<x<C p x+\gamma+w / 2, C p y-w / 2<y<$

$C p y+w / 2\}, \quad P_{w}^{r e f 2}=\{I(x, y) \mid C p x-\gamma-w / 2<x<$ $C p x-\gamma+w / 2, C p y-w / 2<y<C p y+w / 2\}$, and $\gamma=0.5 * r_{r i}+r_{p}$. The $\kappa$ is a constant (bias factor) and values of $\kappa=1.5$ and $\mathrm{w}=9$ are found appropriate. A sample filtered image, $E_{\text {adapt }}^{\text {roi }}$ is shown in Fig $1(\mathrm{Q} 1)$. D is a similarity measurement function, which is given as follows to compute distance between patches; $P^{i}, P^{j}$ -

$$
D\left(P^{i}, P^{j}\right)=\left\|w_{i}-w_{j}\right\|_{2}^{2}
$$

To further reduce unwanted pixels from $E_{\text {adapt }}^{\text {roi }}$ we developed two arc shaped kernels (arched structuring elements) $S_{\text {arc1 }}$ and $S_{\text {arc2 }}$ (shown in Fig. 1(R), which are applied to perform morphological erosion on upper part $E_{\text {adapt }}^{\text {up }}=\left\{E_{\text {adapt }}^{\text {roi }}(x, y) \mid 1<y<C p y\right\}$, and lower part $E_{\text {adapt }}^{\text {lo }}=\left\{E_{\text {adapt }}^{\text {roi }}(x, y) \mid C p y<y<N\right\}$, respectively and refined image is obtained as-

$$
E_{\text {adapt }}^{\text {refined }}=\left(E_{\text {adapt }}^{\text {lo }} \text { } S_{\text {arc1 } 1}\right) \cup\left(E_{\text {adapt }}^{\text {up }} \text { } S_{\text {arc2 }}\right)
$$

The underlying idea for morphological processing with proposed structuring elements is that in image obtained in (16) eyelids take curved shape and different than shape of other objects in the image. Thus, during (18), only eyelid candidate pixels survive and other artifacts are either eliminated or atleast their effect is reduced (Fig. 1(S)).

In second tier of the process, using Hough transform, parabolas of the form, $y=a(x-b)^{2}+c$, are detected for refined edge point set of lower and upper eyelids respectively. In addition, during parabola detection, we employed two constraints to further minimize computation; i) convex and concave shape assumption for upper and lower eyelids, ii) the parameter a, which controls the curvature, is restricted in the interval- $[0.0005,0.002]$. The search of parabola is performed by varying the value of a in the interval with increment of $\operatorname{step}_{a}(0.0002)$. The other parameters i.e. $b$ and $c$ are optimized by constraining the search region for eyelids during ROI extraction above. Finally, a mask containing pixels between eyelids and with radius $=1.20 * \mathrm{r}_{\text {ri }}$ is created as Mask $k_{\text {eyelid }}$ (Fig. 1(U)).

\subsection{Refinement of iris outer boundary}

To obtain refined iris outer boundary, we first confine the search region to detect limbic boundary points using the value of rough iris width $\left(\mathrm{r}_{\mathrm{ri}}\right)$ obtained in section 2.3. In the process, an image $I_{\text {adapt }}$ is created by adaptively thresholding pixels which fall inside a circular stripped region $\left(\mathrm{R}_{\mathrm{cs}}\right)$ in $\mathrm{I}(\mathrm{x}, \mathrm{y})$. The region $R_{c s}($ Fig. $1(\mathrm{~V}))$ is taken with respect to pupil center and can be defined as, $R_{c s}=\{(x, y) \mid x=C p x+r \cos \theta, y=C p y+r \sin \theta$, $0.90 * r_{r i} \leq r \leq 1.10 * r_{r i}$. The thresholding procedure that follows is the same as employed by us in (9) above and thus details are omitted here. The parameters are taken as, $\mathrm{w}=15$ and $\Delta \mathrm{c}=5$, which are empirically determined. A sample $\mathrm{I}_{\text {adapt }}$ is shown in Fig. 1(W).

Next, in $\mathrm{I}_{\text {adapt }}$ the pixels which falls outside upper eyelid and lower eyelid are set 0 , using $M a s k_{\text {eyelid, given as, }}$ $I_{\text {adapt }}^{1}=I_{\text {adapt }} \cap$ Mask $_{\text {eyelid }}$. Further, Canny edge detector is applied on image $\mathrm{I}(\mathrm{x}, \mathrm{y})$, which generates 
edgemap, $\mathrm{I}_{\text {canny }}$ (Fig. 1(X1)). Then the pixels which have value 1 in $I_{\text {canny }}$ and $I_{\text {adapt }}^{2}$ both are extracted as refined candidate iris boundary points to model limbic boundary. The output image is defined as $I_{\text {ipts }}$ (Fig. 1(X2)).

Since, iris boundary may not be perfectly circular or elliptical, in our approach, we propose, to model left and right side iris boundary separately. More specifically, we applied circular Hough transform separately, for the left and right side iris candidate points in $I_{i p t s}$ image. The radius of both the circles is restricted within the interval $\left[0.9 r_{r i}, 1.1 r_{r i}\right]$. (Fig. 1(Y)). To create final iris mask, we generate Mask $_{l r c}$ by setting the values of all pixels inside both circles to 1 and 0 elsewhere. The values corresponding to pupil mask $\left(\operatorname{Mask}_{p}\right)$ are set to 0 . The common pixels with value 1 between Mask $k_{l r c}$ and Mask eyelid are then extracted as final iris mask defined as Mask $_{\text {iris }}=$ Mask $_{\text {lrc }} \cap$ Mask $_{\text {eyelid }}$ (Fig. 1(Z1)).

\section{Experiments and Results}

\subsection{Experiment settings}

A series of experiments have been carried out to evaluate the performance of the proposed framework using four publicly available large scale challenging databases; IITD [21], Casia Interval v4 [22], ND-IRIS-0405 [23], and Face and Ocular Challenge Series (FOCS) [20]. Each database contains images with real-world conditions occur that may be challenging for iris systems. The FOCS has most challening images among them were extracted from near infrared (NIR) video sequences collected from the Iris on the Move (IoM) system. All the experiments are implemented using Matlab 7.0 on an Intel Pentium 3.0 GHz processor PC with 8 GB RAM. During segmentation, the same settings as mentioned in respective subsections above are used for all the datasets for proposed approach wherese database specific parameters (i.e. range of pupil and limbic radious) are used for all other approaches as suggested in other works $[18,19,24]$.

\subsection{Segmentation Performance Evaluation using Ground Truth Data}

In order to evaluate the segmentation performance of our approach, we utilized IRISSEG-EP [8] dataset, which contains manually generated ground truth data for Casia v4 Interval, ND-IRIS-0405, and IITD. In this dataset, at least 5 data points for inner and outer iris circumference and 3 data points for upper and lower eyelids are provided. The iris segmenteation is performed by modeling iris inner and outer boundaries using ellipse fitting and eyelid boundaries by fitting a second order polynomial. During evaluation, the iris mask created by our approach are compared with ground truth mask using well known measures; precision $(\mathrm{P})$, recall $(\mathrm{R})$ and $\mathrm{F}$-measure $(\mathrm{F})$, similar to [8]. The
Table I: THE P, R AND F VALUES FOR THE ALGORITHMIC GENERATED MASKS WHEN COMPARED TO GROUND TRUTH PROVIDED BY OPERATOR A

\begin{tabular}{|c|c|c|c|c|c|c|c|}
\hline \multirow{2}{*}{$D B$} & \multirow{2}{*}{ pproach } & \multicolumn{2}{|c|}{$R$} & \multicolumn{2}{|c|}{$P$} & \multicolumn{2}{|c|}{$F$} \\
\hline & & $\boldsymbol{\mu}$ & $\boldsymbol{\sigma}$ & $\mu$ & $\boldsymbol{\sigma}$ & $\mu$ & $\boldsymbol{\sigma}$ \\
\hline \multirow{8}{*}{ 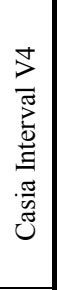 } & GST & 85.19 & 18 & 9.91 & 7.37 & 86.16 & 11. \\
\hline & Osiris & & 7.93 & & & & 5.47 \\
\hline & WAHET & 94.72 & 9.01 & 85.44 & 9.67 & 39.13 & 8.39 \\
\hline & IFFP & 1.74 & 14.74 & & 14.26 & 86.86 & 13.27 \\
\hline & CAHT & 7.68 & 4.56 & 82.89 & 9.95 & 89.27 & 6.67 \\
\hline & Masek & & & & & & 9 \\
\hline & IDO & 1.34 & 22.86 & 61.62 & 18.71 & 65.61 & 19.96 \\
\hline & roposec & 94.26 & 4.18 & 92.15 & 3.34 & 93.10 & 2.65 \\
\hline \multirow{8}{*}{ 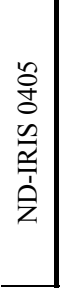 } & $\overline{\text { GST }}$ & 1.07 & 12.57 & 83.72 & 10.83 & 14 & 9.57 \\
\hline & Osiris & 0 & 6.68 & 08 & 3 & & .61 \\
\hline & WAHET & 3.65 & 12.12 & 82.24 & 14.03 & 86.79 & 11.85 \\
\hline & IFFP & 2.62 & 9.83 & 79.55 & 13.93 & & 11.05 \\
\hline & CAHT & 32 & 20.11 & 72.71 & 18.62 & 80.51 & 18.57 \\
\hline & Masek & 7 & 5.43 & 95.29 & 5. & 87 & 11.53 \\
\hline & IDO & .73 & 23.38 & 73.66 & 18.16 & 78.27 & 19.90 \\
\hline & propose & 91.93 & 5.10 & 95.02 & 5.34 & 93.29 & 3.94 \\
\hline \multirow{8}{*}{ 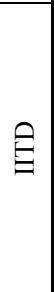 } & GST & 0.06 & 16.65 & 85.86 & 10.46 & 8( & 11.87 \\
\hline & Osiris & .06 & 6.43 & 91.01 & 7.61 & 92.23 & 5.80 \\
\hline & WAHET & 97.43 & 8.12 & 79.42 & 12.41 & 87.02 & 9.72 \\
\hline & IFFP & 93.92 & 10.62 & 79.76 & 11.42 & 85.83 & 9.54 \\
\hline & CAHT & 96.8 & 11.2 & 78.87 & 13.25 & 86.28 & 11.39 \\
\hline & Masek & 6.23 & 18.74 & & & & 15.39 \\
\hline & IDO & 51.91 & 15.32 & 52.23 & 14.85 & 51.17 & 13.26 \\
\hline & proposed & 95.33 & 4.58 & 93.70 & 5.33 & 94.37 & 3.88 \\
\hline
\end{tabular}

produced iris mask is categorised as-true positives (tp), the number of iris pixels which were correctly marked; false positives (fp), the number of non-iris pixels which were marked; false negative (fn), the number of unmarked iris pixels; and true negative (tn), the number of unmarked non-iris pixel. The recall, precision and F-measure are then computed as-

$$
\begin{aligned}
& P=t p /(t p+f p) \\
& R=t p /(t p+f n) \\
& F=2 R P /(R+P)
\end{aligned}
$$

For comparative evaluation of segmentation accuracies, we also utilized other algorithms such as; GST [9], CAHT [7], WAHET [18], Osiris [19], IFFP [10], Libor Masek's implementation [24], and Integro-Differential Operator (IDO) [1]. The segmentation accuracies are reported in Table I. It is illustrated in Table I, that, proposed approach not just provides highest $\mathrm{F}$-measure and lowest variance over the predictions compared to other competitive algorithms, but also it provides significant improvements for F-measure than other methods across all test datasets.

\subsection{Segmentation Performance Evaluation on challenging Periocular Images (FOCS database)}

We choose a subset of 1200 images for comparison purpose randomly selected from 120 classes. Since, no ground truth data is available for FOCS [10], the iris segmentation accuracy is calculated manually by visual 
inspection. We consider segmentation correct when, i) the iris mask generated using proposed framework overlap the true iris region completely and ii) the two circles required for polar conversion are fitted properly and normalization is done accurately. The iris localization accuracy of different approaches is shown in Table II. The reported segmentation accuracy (SA) is computed as-

$$
S A=\frac{\text { No.of correctly segmented images }}{\text { Total images tested }} \times 100
$$

The images in FOCS are very challenging and in many images even the irises are not visible. During visual inspection of images segmented using our approach, we did not find major issues. In few cases, it could not segment properly when images are either severely damaged or iris region is completely merged with sclera. It is clearly seen from Table II, that our approach achieves very promising SA (93.41\%) compared to other methods. Also, our approach reports a significant improvement of $3.11 \%$ in SA over best SA reported on FOCS in literature (90.30\% [13]), even on a much bigger dataset.

\subsection{Recognition Performance and Running Time}

We utilized the IRISSEG-EP ground truth data [8] as baseline segmentation approach. Fig. 2-5 illustrates the ROC performance of our segmentation framework compared with baseline, classical methods such as Libor Masek's [24], and IDO [1] and other recently published competitive methods; CAHT, WAHET, Osiris, GST and IFFP. The EER of the approaches is reported in Table III. To compute verification accuracies, we adopted a simple iris recognition pipeline, in which, for normalization, we used Daugman's rubber sheet model [1] and all irises are normalized into rectangle of $64-$ by-512 pixels. For iris feature extraction, 1-D Log-Gabor filter with the parameters (same for all our experiments) as in [5] is used and the similarity score is computed using Hamming distance between shifted templates [1]. Since, the main focus of this paper is to propose a segmentation framework, we did not explore other state of the art approaches for feature extraction, postprocessing, normalization or classification etc.. To do a fair comparison, we applied the same steps for all the methods after segmentation stage. Due to this, there may be some deviations in accuracy reported in this work and accuracy reported in original publication of respective method or accuracies reported on these databases in some other published work.

From Table III, it is clear that the proposed framework achieves best verification rates (i.e. EER) compared to other algorithms on all test datasets. On the other hand, the other approaches do not show consistency in their performance, such as Osiris and GST perform well on IITD and ND-iris, but not so well on Casia Interval and FOCS. WAHET performs quite well on Casia Interval but performs badly on ND-iris, IITD and FOCS. CAHT performs well on IITD and CASIA Interval but could not
Table II: Segmentation Accuracy on FOCS Dataset

\begin{tabular}{c|ccc}
\hline Approach & $\begin{array}{c}\text { Input } \\
\text { Images }\end{array}$ & $\begin{array}{c}\text { No. of Correctly } \\
\text { Segmeneted Images }\end{array}$ & $\begin{array}{c}\text { Segmentation } \\
\text { Accuracy (\%) }\end{array}$ \\
\hline Osiris [19] & 1200 & 817 & 68.12 \\
GST [9] & 1200 & 650 & 54.12 \\
WAHET [18] & 1200 & 906 & 75.49 \\
IFFP [20] & 1200 & 709 & 59.12 \\
CAHT [7] & 1200 & 851 & 71.00 \\
Proposed & 1200 & 1120 & $\mathbf{9 3 . 4 1}$ \\
Masek [24] & 1200 & 635 & 52.92 \\
IDO [1] & 1200 & 781 & 65.08 \\
GAC [13]* & 404 & 358 & 88.60 \\
Hough Transform [13]* & 404 & 210 & 52.00 \\
Active Contours & 404 & 365 & 90.30 \\
Without Edges [13]* & & & \\
\hline Rop
\end{tabular}

*Reported in original publication [13]

Table III: Verification Accuracy (EER in \%)

\begin{tabular}{c|cccc}
\hline Approach & IITD & ND-IRIS 0405 & CASIA Interval V4 & FOCS \\
\hline Osiris & 1.38 & 3.18 & 6.36 & 26.27 \\
WAHET & 7.44 & 7.72 & 1.24 & 27.11 \\
IFFP & 5.88 & 15.56 & 7.24 & 38.41 \\
CAHT & 0.68 & 8.53 & 0.78 & 27.41 \\
GST & 0.90 & 2.84 & 4.44 & 35.12 \\
Proposed & 0.50 & 2.31 & 0.62 & 21.11 \\
\hline Groundtruth & 0.18 & 1.54 & 0.57 & -- \\
Masek & 13.54 & 13.72 & 5.75 & 35.52 \\
IDO & 27.36 & 17.71 & 36.45 & 26.36 \\
\hline
\end{tabular}

Table IV: Average Execution Per image (second)

\begin{tabular}{c|c|c|c|c|c|c|c|c|c}
\hline Approach & IFFP & CAHT & WAHET & Osiris & GST & Masek & IDO & Proposed \\
\hline Implemented & \multicolumn{5}{|c}{$C / C++$} & & \multicolumn{5}{c}{ MATLAB } \\
\hline Exec. time & 0.40 & 2.2 & 0.30 & 5 & 24 & 11 & 10 & 3.4 \\
\hline
\end{tabular}

do well on ND-iris and FOCS. The IDO, Libor Masek's implementation and IFFP show comparatively very discouraging results on all datasets. On FOCS, which contains the most challenging images, our approach obtains far better results and a significant improvement in EER i.e. $5 \%$ to $17 \%$ over other methods.

From experimental study, it can be concluded that the proposed approach not only provides impressive performance even on challenging images, but it also generalizes well compared to other state-of-the-art methods. Furthermore, an analysis of ROCs on CASIA Interval V4, ND-IRIS-0405, and IITD datasets also reveals that, proposed approach reports accuracy close to the accuracy obtained on the images segmented using groundtruth data. Such encouraging performance consistently across all datasets, further justifies the validity and superiority of the proposed segmentation framework.

The average running time of the approaches is reported in Table IV. Our proposed approach without doubt runs faster than other methods which are implemented in MATLAB. In our approach, the improvement in execution time is certainly because of the employed proposed less complex procedures during various stages in iris segmentation. The major reason for relatively lesser execution time shown by the methods implemented in 


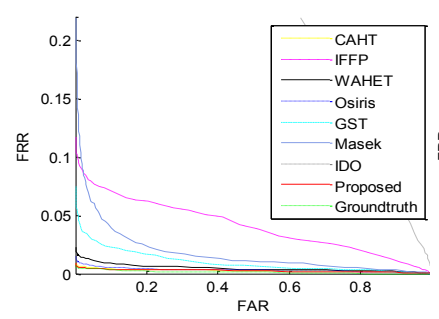

Fig. 2: ROC for Casia Interval V4

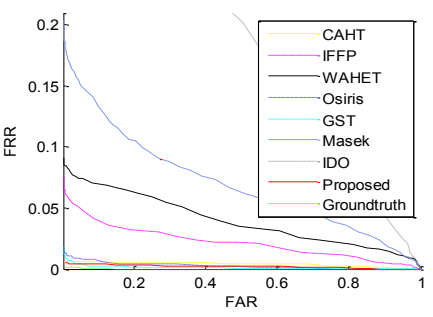

Fig. 3: ROC for IITD

$\mathrm{C} / \mathrm{C}++$ is that $\mathrm{C} / \mathrm{C}++$ generates relatively optimized machine code compared to MATLAB.

\section{Conclusion and Future work}

This paper has presented a robust iris segmentation framework designed specifically for non-ideal irises. The proposed approach is based on adaptive filtering and follows a coarse-to-fine strategy. We propose various novel and efficient procedures specifically for pupil, limbic and eyelid boundary detection. The approach exploits proposed local features and fusion of their scores for robust pupil localization. Considering the heterogeneity of databases, the approach also presents dynamic computation of parameters required during various stages in segmentation. Further, a comparative experimental analysis among our approach, segmentation using groundthruth data, classical approaches such as Libor Masek's and Integro-Differential Operator along with modern approaches such as CAHT, IFFP, WAHET, Osiris, and GST over challenging databases such as Casia Interval V4, ND-IRIS-0405, IITD and FOCS is conducted. The results demonstrate superior performance of the proposed framework. It is also shown from results that the proposed approach has better generalization capabilities compared to other methods.

The experimental study in this paper has been limited to NIR iris images only, thus future work will focus on adaptation of the approach to visible spectrum images.

\section{References}

[1] J. Daugman, "How Iris Recognition Works", IEEE Trans. Circuits and Systems for Video Technology, vol. 14, no. 1, pp. 21-30, Jan. 2002.

[2] R. Wildes. Iris recognition: an emerging biometric technology. Proceedings of the IEEE, Vol. 85, No. 9, 1997.

[3] T. Tan, Z. He, and Z. Sun, "Efficient and robust Seg. of noisy Iris images for non-cooperative Iris recognition," Image Vis. Comput., vol. 28, no. 2, pp. 223-230, 2010.

[4] He, Z., Tan, T., Sun, Z., Qiu, X.: 'Toward accurate and fast iris segmentation for iris biometrics', IEEE Trans. Pattern Anal. Mach. Intell., 2009, 31, (9), pp. 1670-1684.

[5] C.-W. Tan and A. Kumar, "A unified framework for automated Iris segmentation using distantly acquired face images," IEEE Trans. Image Process., vol. 21, no. 9, pp. 4068-4079, Sep. 2012.

[6] Y.-H. Li, M. Savvides, An Automatic Iris Occlusion Estimation Method Based on High-Dimensional Density

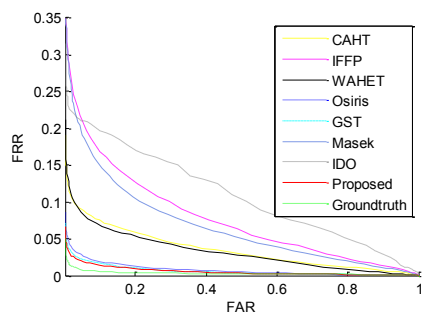

Fig. 4: ROC for ND-IRIS-0405

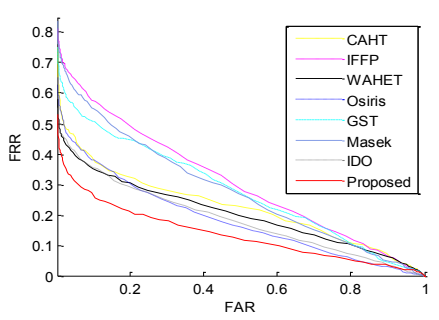

Fig. 5: ROC for FOCS
Estimation, IEEE Transactions on Pattern Analysis and Machine Intelligence 35 (4) (2013) 784-796.

[7] C. Rathgeb, A. Uhl, and P. Wild, Iris Recognition: From Segmentation to Template Security, ser. Advances in Information Security. Springer Verlag, 2013, vol. 59.

[8] Heinz Hofbauer, Fernando Alonso-Fernandez, Peter Wild, Josef Bigun and Andreas Uhl, "A Ground Truth for Iris Segmentation", Proc. 22nd International Conference on Pattern Recognition, ICPR, Stockholm, Aug. 24-28, 2014.

[9] F. Alonso-Fernandez, J. Bigun, "Iris boundaries Seg. using the generalized structure tensor.," Proc. BTAS, 2012.

[10] A. Uhl and P. Wild, "Multi-stage visible wavelength and near infrared iris segmentation framework," in Proceedings of the International Conference on Image Analysis and Recognition (ICIAR'12), ser. LNCS, 2012, pp. 1-10.

[11] J. Daugman, "New Methods in Iris Recognition", IEEE Trans. System, Man,and Cybernetics-Part B: Cybernetics, vol. 37, no. 5, pp. 1167-1175, 2007.

[12] Shah, S., Ross, A.,: Iris Segmentation Using Geodesic Active Contours, IEEE Transactions on Information Forensics and Security (TIFS), vol. 4, no. 4, pp. 824-836, Dec. (2009).

[13] Jillela, R., Ross, A., Boddeti, N., Vijaya Kumar, B., Hu, X., Plemmons, R., Pauca, P.: An Evaluation of Iris Segmentation Algorithms in Challenging Periocular Images, Chapter in Handbook of Iris Recognition, 2013

[14] Akanksha Joshi, Abhishek Gangwar, Zia Saquib "Person recognition based on fusion of iris and periocular biometrics," 12th International Conference on Hybrid Intelligent Systems (HIS), 2012.

[15] H. Li, Z. Sun, T. Tan, Robust iris segmentation based on learned boundary detectors, in: 5th IAPR International Conference on Biometrics, 2012, pp. 317-322.

[16] E. Ortiz, K. Bowyer, P. Flynn, An optimal strategy for dilation based iris image enrollment, in: IEEE International Joint Conference on Biometrics, 2014, pp. 1-6.

[17] Chun-Wei Tan and Ajay Kumar, "Towards online iris and periocular recognition under relaxed imaging constraints," IEEE Trans Image Process. 2013 Oct;22(10):3751-65.

[18] A. Uhl and P. Wild, "Weighted Adaptive Hough and Ellipsopolar transforms for real-time iris segmentation," in Proceedings of the 5th IAPR/IEEE Inte rnational Conference on Biometrics (ICB'12), 2012, pp. 1-8.

[19] D. Petrovska and A. Mayoue, "Description and documentation of the biosecure software library," Project No IST-2002-507634 - BioSecure, Deliverable, 2007.

[20] http://www.nist.gov/itl/iad/ig/focs.cfm

[21] http://web.iitd.ac.in/ biometrics/Database_Iris.htm

[22] http://biometrics.idealtest.org

[23] http://www3.nd.edu/ cvrl/CVRL/Data_Sets.html

[24] http://www.peterkovesi.com/studentprojects/libor/sourcecod e.html 